\title{
Parámetros de Interacción lónica de las Soluciones Acuosas Concentradas del Líquido lónico Trifluorometanosulfonato de 1-Etil-3-Metil-Imidazolio a Varias Temperaturas
}

\author{
Manuel S. Páez ${ }^{(1) \star}$, Jesús A. Miranda ${ }^{(1)}$, Francisco J. Torres ${ }^{(2)}$ \\ (1) Universidad de Córdoba. Departamento de Química. Facultad de Ciencias Básicas. \\ Carrera 6 No. 76-103. Km 3. Vía Cereté. Córdoba. Colombia. (e-mail: mspaezm@unal.edu.co.) \\ (2) Universidad de Córdoba. Departamento de Física. Facultad de Ciencias Básicas. Carrera 6 No. 76 - \\ 103. Km 3. Vía Cereté. Córdoba. Colombia. \\ * Autor a quien debe dirigirse la correspondencia.
}

Recibido Mar. 31, 2014; Aceptado Jun. 19, 2014; Versión final recibida Jul. 9, 2014

\begin{abstract}
Resumen
Se determinaron experimentalmente las densidades de las soluciones acuosas concentradas de la mezcla binaria agua + trifluorometanosulfonato de 1-etil-3-metil-imidazolio ([Emim+] $\left[\mathrm{CF}_{3} \mathrm{SO}_{3}{ }^{-}\right]$) a las temperaturas de 283.15 a $318.15 \mathrm{~K}$, usando un densímetro de tubo vibratorio Anton Paar modelo DMA 5000. Se calcularon los volúmenes molares aparentes y se correlacionaron con las ecuaciones de PitzerSimomson usando como estado de referencia al líquido iónico puro y el soluto a dilución infinita, y con los modelos de Pitzer-Simomson-Clegg y Pitzer-Simomson-Clegg simplificado, cuyo estado de referencia es el líquido iónico puro. El coeficiente de correlación resultó positivo y cercano a uno, lo cual indica que existe una correlación directa, entre los volúmenes molares aparentes experimentales y calculados para todos los casos. Sin embargo, los resultados de la correlación muestran que los volumenes molares aparentes se ajustan mejor a la ecuación de Pitzer-Simomson.
\end{abstract}

Palabras clave: densidad, trifluorometanosulfonato de 1-etil-3-metil-imidazolio, volumen molar aparente, ecuación de Pitzer-Simomson, ecuación de Pitzer-Simomson-Clegg.

\section{Ionic Interaction Parameters of Concentrated Aqueous Solutions of the Ionic Liquid 1-Ethyl-3-Methyl-Imidazolium Trifluoromethanesulfonate at Various Temperatures}

\begin{abstract}
Densities of concentrated aqueous solutions of the binary mixture water +1 -ethyl-3-methyl-imidazolium trifluoromethanesulfonate $\left(\left[\mathrm{Emim}^{+}\right]\left[\mathrm{CF}_{3} \mathrm{SO}_{3}{ }^{-}\right]\right)$were experimentally determined at temperatures from 283.15 to $318.15 \mathrm{~K}$, using an Anton Paar model DMA 5000 vibrating tube densitometer. Apparent molar volumes were calculated and correlated with the Pitzer-Simomson equation using as reference state the pure ionic liquid and the solute at infinite dilution and with the Pitzer-Simomson-Clegg and SimplifiedPitzer-Simomson-Clegg models whose reference state is the pure ionic liquid. The correlation coefficient was positive and close to one, indicating that there is a direct correlation, between the experimental and calculated apparent molar volumes for all cases. However, the correlation results show that the apparent molar volumes fit better to the Pitzer-Simomson equation.
\end{abstract}

Keywords: density, 1-ethyl-3-methyl-imidazolium trifluoromethanesulfonate, apparent molar volume, Pitzer-Simonson equation; Pitzer-Simomson-Clegg equation 


\section{INTRODUCCIÓN}

Los modelos de interacción de soluciones acuosas de electrolitos (Wijesinghe et al., 2005; Kodejš et al., 1986; Galleguillos et al., 1998; Páez et al., 2013) proporcionan un método fácil y adecuado para el cálculo de diversas cantidades volumétricas. Sin embargo, estos modelos por su empirismo implícito en su desarrollo, requieren la evaluación de los parámetros de interacción a partir de datos experimentales (Rowland et al., 2013; Song, 2010), afortunadamente Pitzer y Simomson desarrollaron un modelo alternativo basado en fracción molar (Pitzer et al., 1986). Luego, Clegg y Pitzer (1992) extendieron el modelo introduciendo términos dependientes de la concentración en la expresión de Debye-Hückel y un parámetro adicional de corto alcance para la interacción entre el solvente y un anión y un catión en soluciones altamente concentradas, estos se han usado con éxito en la correlación del volumen molar aparente de algunos líquidos iónicos con diversos solventes (Yang et al., 2005; Tong et al., 2009).

Sin embargo, debido a que no se han encontrado estudios del comportamiento volumétrico del líquido iónico $\left[\mathrm{Emim}^{+}\right]\left[\mathrm{CF}_{3} \mathrm{SO}_{3}{ }^{-}\right]$en agua, que realicen este tipo de correlaciones, es necesario realizar el estudio del mismo; por tal razón, en el presente trabajo se reportan las densidades de las soluciones acuosas de [Emim+][ $\left.\mathrm{CF}_{3} \mathrm{SO}_{3}{ }^{-}\right]$, en el intervalo de concentraciones [0.0417 - 0.9583] en la escala de fracción molar, a las temperaturas de $(283.15$ a 318.15$) \mathrm{K}$, con intervalos de $5 \mathrm{~K}$, y a partir de estas, se calcularon los volúmenes molares aparentes, ${ }_{1}^{\emptyset} V$ y se correlacionaron con los modelos PS, PSC y PSC simplificada, tomando como estado de referencia el líquido iónico puro y el soluto a dilución infinita para el primero; mientras que para el segundo se tomó el líquido iónico puro.

\section{FUNDAMENTOS TEÓRICOS}

El desarrollo de modelos termodinámicos para soluciones de electrolitos debe considerar al menos dos efectos, uno relacionado con las fuerzas de largo alcance entre las especies iónicas y otro debido a fuerzas de corto alcance entre molécula-molécula y molécula- especies iónicas (Song, 2010).

\section{Ecuación de Pitzer-Simomson (PS).}

Pitzer y Simomson (1986) desarrollaron un modelo termodinámico basado en la escala de fracción molar, para mezclas que contienen iones de tipo carga simétrica, que es aplicable en todo el intervalo de concentración. Este tratamiento asume que la energía libre de Gibbs de exceso de la mezcla consiste de dos términos: Uno que toma en cuenta las fuerzas de corto alcance expresado por una expansión de Margules en función de la fracción molar, y otro término de largo alcance dado por Debye-Hückel. Siguiendo este razonamiento, la ecuación de trabajo de PS para volumen molar aparente ${ }_{1}^{\phi} V$, tomando en cuenta como estado de referencia el líquido iónico puro puede ser expresada como (Tong et al., 2009).

$Y_{1}=\left\{{ }_{1}^{\emptyset} V-V_{L I}^{0}-\left(A_{x}^{V} / b\right) \operatorname{Ln}\left[\left(1+b I_{x}^{1 / 2}\right) /\left(1+b I_{x}^{01 / 2}\right)\right]\right\} / 2 R T x_{1}=W_{1, M X}^{V}+x_{2} U_{1, M X}^{V}$

Mientras que si se toma como estado de referencia el soluto a dilución infinita, la ecuación de trabajo para la determinación de los parámetros volumétricos de PS viene dada por (Yang et al., 2005).

$$
Y_{2}=\left\{{ }_{1}^{\emptyset} V-\left(A_{x}^{V} / b\right) \operatorname{Ln}\left(1+b I_{x}^{1 / 2}\right)\right\} / 2 R T=\bar{V}_{L I}^{0} / 2 R T+x_{2}\left[W_{1, M X}^{V}+x_{1} U_{1, M X}^{V}\right]=a_{0}+a_{1} x_{2}+a_{2} x_{1} x_{2}
$$

Donde $V_{L I}^{0}$, es el volumen molar del líquido iónico puro, $\bar{V}_{L I}^{0}$ es el volumen molar parcial a dilución infinita, $A_{x}^{V}$ es el parámetro de Debye-Hückel, $I_{x}$ es la fuerza iónica de la mezcla, ambos en la escala de fracción molar, $I_{x}^{0}$ es la fuerza iónica del líquido iónico puro, la cual toma el valor de $1 / 2$ para líquidos iónicos de tipo 1:1. La fuerza iónica viene dada por $I_{X}=(1 / 2) \sum x_{i} Z_{i}^{2}=x_{M}=x_{X}=\left(1-x_{1}\right) / 2$ y $x_{2}=x_{M}+x_{X}=\left(n_{X}+n_{M}\right) /\left(n_{\text {agua }}+\right.$ $\left.n_{M}+n_{X}\right)_{0}=2 n_{L I} /\left(n_{\text {agua }}+2 n_{L I}\right)$ y $x_{1}=x_{\text {agua }}=n_{\text {agua }} /\left(n_{\text {agua }}+2 n_{L I}\right)$, aquí $x_{i}=n_{i} /\left(n_{1}+2 n_{i}\right)$. Los términos $W_{1, M X}^{V}$ y $U_{1, M X}^{V}$ son parámetros ajustables y dependen del estado de referencia, temperatura y presión, ellos a su vez son característicos para cada soluto $M X$.

\section{Ecuación de Pitzer-Simomson-Clegg (PSC).}

Clegg y Pitzer (1992) extendieron el modelo de Pitzer y -Simomson (1986) introduciendo términos dependientes de la concentración en la expresión de Debye-Hückel y un parámetro adicional de corto alcance, $B_{M X} g(x)$ para describir la interacción del solvente con un anión y un catión en soluciones altamente concentradas. De acuerdo con la teoría de PSC la ecuación de trabajo teniendo como estado de referencia el líquido iónico puro viene dada por (Tong et al., 2009). 
$Y_{3}=\left\{{ }_{1}^{\emptyset} V-V_{L I}^{0}-\left(A_{x}^{V} / b\right) \operatorname{Ln}\left[\left(1+b I_{x}^{1 / 2}\right) /\left(1+b I_{x}^{01 / 2}\right)\right]\right\} / 2 R T x_{1}=W_{1, M X}^{V}+x_{2} U_{1, M X}^{V}+x_{1} x_{2} V_{1, M X}^{V}+x_{2}^{2} B_{M X}^{V}\left(2 I_{x}^{1 / 2}\right)$

Donde $V_{1, M X}^{V}$ y $B_{M X}^{V}$ son los nuevos parámetros de la teoría de PSC.

Ecuación de Pitzer-Simomson-Clegg simplificada (PSCS).

Zhang et al. (1986) indican que el término que contiene al parámetro $B_{M X}^{V}$ es tomado por analogía con el modelo de Pitzer basado en la escala de molalidad y puede ser visto como un coeficiente de la fuerza iónica dependiente de la adición del término de Debye-Hückel. Sin embargo, el parámetro $B_{M X}^{V}$ en la ecuación (3) puede ser despreciado para soluciones diluidas, por consiguiente si se toma en cuenta el líquido iónico puro como estado de referencia, la ecuación de PSC se reduce a la expresión (Tong et al., 2009).

$Y_{4}=\left\{{ }_{1}^{\varnothing} V-V_{L I}^{0}-\left(A_{x}^{V} / b\right) \operatorname{Ln}\left[\left(1+b I_{x}^{1 / 2}\right) /\left(1+b I_{x}^{01 / 2}\right)\right]\right\} / 2 R T x_{1}=W_{1, M X}^{V}+x_{2} U_{1, M X}^{V}+x_{1} x_{2} V_{1, M X}^{V}$

\section{MATERIALES Y MÉTODOS}

El líquido iónico trifluorometanosulfonato de 1-etil-3-metil-imidazolio fué obtenido de Sigma Aldrich con una pureza $\geq 99 \%$. Para reducir el contenido de agua y los compuestos volátiles a valores insignificantes, este LI se secó con agitación a temperatura moderada $(T=343 \mathrm{~K})$ y bajo vacío $(\mathrm{p}=0,2 \mathrm{~Pa})$ aproximadamente durante $48 \mathrm{~h}$ antes de su uso. Después del secado se guardó en una botella bajo una atmósfera de gas inerte. Luego se midió el contenido de agua de este líquido iónico usando un Coulómetro Karl Fisher (Metrohm 831). En este proceso, el yodo se genera con precisión eléctricamente en la solución de Karl Fisher y el contenido de agua se determina por la cantidad de yodo que reacciona con el agua en una relación de uno a uno. Para este propósito se usó un tamaño de muestra de aproximadamente 0,1 gramos para cada medición, y se repitió dos veces para cada muestra, dando como resultado un contenido de agua de 100ppm. El agua usada para la calibración y preparación de las soluciones fue bidestilada y desgasificada y presentó una conductividad menor que $2 \mu \mathrm{S}$. La preparación de las soluciones del sistema acuoso $\left[\mathrm{Emim}^{+}\right]\left[\mathrm{CF}_{3} \mathrm{SO}_{3}{ }^{-}\right]$se realizó mediante el método gravimétrico usando una balanza OHAUS con incertidumbre de $\pm 1 \times 10^{-4} \mathrm{~g}$ en botellas herméticamente selladas para minimizar pérdidas por evaporación. La densidad del líquido puro y las soluciones se midió usando un densímetro de tubo vibratorio marca Anton Paar modelo DMA 5000 con una incertidumbre de $\pm 1 \times 10^{-5} \mathrm{~g} / \mathrm{cm}^{3}$.

\section{RESULTADOS Y DISCUSIONES}

Los valores de densidad $(\rho)$ para el líquido iónico puro y las soluciones acuosas del líquido iónico $\left[\mathrm{Emim}^{+}\right]\left[\mathrm{CF}_{3} \mathrm{SO}_{3}{ }^{-}\right]$se determinaron en el intervalo de temperaturas [283.15-318.15] $\mathrm{K}$ y se muestran en las Tablas 1 y 3 respectivamente. En la Tabla 1, se comparan las medidas de densidad del [Emim+] $\left.\mathrm{CF}_{3} \mathrm{SO}_{3}{ }^{-}\right]$ puro obtenidas en este trabajo, con los datos de literatura ( ${ }^{\star}$ Este trabajo; aVercher et al., 2007; bRodríguez et al., 2006), observándose que son coincidentes. Esta afirmación está sustentada en los resultados obtenidos con la aplicación de la prueba $t$-student para muestras apareadas (Miller et al., 2002) tal como se muestra en la Tabla 2. Dado que $t$ crítico $\left(t_{c}\right)$ es mayor que $t$ calculado ( $\left.t_{c a l}\right)$ se concluye que no existe diferencia significativa para el valor de la densidad obtenida y los reportados en la literatura.

Tabla 1: Densidades de [Emim+] $\left[\mathrm{CF}_{3} \mathrm{SO}_{3}{ }^{-}\right]$puro comparados con valores de literatura en el intervalo de temperatura de $283.15-318.15 \mathrm{~K}$

\begin{tabular}{ccccccccc}
\hline \multicolumn{7}{c}{$\rho /{\mathrm{g} . \mathrm{cm}^{-3}}^{-3}$} \\
\hline $\mathrm{T} / \mathrm{K}$ & 283.15 & 288.15 & 293.15 & 298.15 & 303.15 & 308.15 & 313.15 & 318.15 \\
\hline$\left[\mathrm{Emim}^{+}\right]\left[\mathrm{CF}_{3} \mathrm{SO}_{3}{ }^{-}\right]$ & $1.39168^{*}$ & $1.39222^{*}$ & $1.38797^{*}$ & $1.38463^{*}$ & $1.38343^{*}$ & $1.37824^{*}$ & $1.37302^{*}$ & $1.36959^{*}$ \\
& $1.39626^{\mathrm{a}}$ & $1.39202^{\mathrm{a}}$ & $1.38779^{\mathrm{a}}$ & $1.38358^{\mathrm{a}}$ & $1.37938^{\mathrm{a}}$ & $1.37519^{\mathrm{a}}$ & $1.37101^{\mathrm{a}}$ & $1.36685^{\mathrm{a}}$ \\
& & & & $1.38360^{\mathrm{b}}$ & & $1.37522^{\mathrm{b}}$ & & $1.36690^{\mathrm{b}}$ \\
\hline
\end{tabular}

Tabla 2: Valores de la prueba $t$-student para comparar las densidades del líquido iónico puro experimentales y los de la literatura.

\begin{tabular}{lccccc}
\hline $\mathrm{P}=0.05 / 2$ colas & $\mathrm{d}_{\text {media }}$ & $\mathrm{S}_{\mathrm{d}}$ & $t_{\text {cal }}$ & $t_{\mathrm{c}}$ & $\mathrm{gl}$ \\
\hline Vercher et al & 0.0011 & 0.0027 & 1.1516 & 2.3646 & 7 \\
Rodríguez et al & 0.0022 & 0.0011 & 3.6487 & 4.3027 & 2 \\
\hline
\end{tabular}


En la Tabla 3, se muestran los resultados experimentales de la densidad del sistema $\left[\mathrm{Emim}^{+}\right]\left[\mathrm{CF}_{3} \mathrm{SO}_{3}{ }^{-}\right]+$ agua, observándose que estos disminuyen con el aumento de la temperatura y aumentan con la concentración del líquido iónico.

Tabla 3: Densidades de las soluciones de $\left[\mathrm{Emim}^{+}\right]\left[\mathrm{CF}_{3} \mathrm{SO}_{3}{ }^{-}\right]+$agua a temperaturas de $283.15 \mathrm{~K}$ a $318.15 \mathrm{~K}$

\begin{tabular}{ccccccccc}
\hline $\mathrm{T} / \mathrm{K}$ & 283.15 & 288.15 & 293.15 & 298.15 & 303.15 & 308.15 & 313.15 & 318.15 \\
\hline $\mathrm{XLI}$ & & & & $\rho / g . \mathrm{cm}^{-3}$ & & & & \\
\hline 0.0000 & 0.99970 & 0.99919 & 0.99829 & 0.99713 & 0.99571 & 0.99408 & 0.99225 & 0.99022 \\
0.0417 & 1.12583 & 1.12388 & 1.12143 & 1.11852 & 1.11640 & 1.11281 & 1.11009 & 1.10625 \\
0.0860 & 1.19842 & 1.19596 & 1.19260 & 1.18913 & 1.18625 & 1.18206 & 1.17875 & 1.17423 \\
0.1250 & 1.23858 & 1.23603 & 1.23224 & 1.22837 & 1.22537 & 1.22068 & 1.21702 & 1.21244 \\
0.1667 & 1.26847 & 1.26599 & 1.26191 & 1.25777 & 1.25480 & 1.24984 & 1.24550 & 1.24142 \\
0.2065 & 1.28934 & 1.28704 & 1.28282 & 1.27854 & 1.27566 & 1.27053 & 1.26582 & 1.26204 \\
0.2500 & 1.30666 & 1.30459 & 1.30029 & 1.29594 & 1.29319 & 1.28794 & 1.28322 & 1.27944 \\
0.2917 & 1.31962 & 1.31779 & 1.31344 & 1.30908 & 1.30646 & 1.30114 & 1.29642 & 1.29267 \\
0.3333 & 1.33012 & 1.32850 & 1.32410 & 1.31979 & 1.31728 & 1.31193 & 1.30721 & 1.30350 \\
0.3750 & 1.33877 & 1.33737 & 1.33296 & 1.32868 & 1.32629 & 1.32092 & 1.31621 & 1.31254 \\
0.4167 & 1.34602 & 1.34484 & 1.34043 & 1.33620 & 1.33392 & 1.32854 & 1.32385 & 1.32021 \\
0.4583 & 1.35220 & 1.35123 & 1.34684 & 1.34266 & 1.34047 & 1.33510 & 1.33041 & 1.32680 \\
0.5000 & 1.35753 & 1.35676 & 1.35238 & 1.34827 & 1.34617 & 1.34080 & 1.33613 & 1.33253 \\
0.5417 & 1.36219 & 1.36159 & 1.35725 & 1.35319 & 1.35117 & 1.34581 & 1.34114 & 1.33757 \\
0.5833 & 1.36629 & 1.36587 & 1.36155 & 1.35755 & 1.35561 & 1.35027 & 1.34561 & 1.34204 \\
0.6250 & 1.36994 & 1.36968 & 1.36539 & 1.36145 & 1.35958 & 1.35424 & 1.34958 & 1.34604 \\
0.6667 & 1.37322 & 1.37310 & 1.36884 & 1.36496 & 1.36316 & 1.35782 & 1.35318 & 1.34964 \\
0.7083 & 1.37617 & 1.37620 & 1.37197 & 1.36814 & 1.36641 & 1.36108 & 1.35643 & 1.35290 \\
0.7500 & 1.37886 & 1.37901 & 1.37482 & 1.37105 & 1.36937 & 1.36405 & 1.35940 & 1.35588 \\
0.7917 & 1.38132 & 1.38160 & 1.37743 & 1.37373 & 1.37210 & 1.36679 & 1.36212 & 1.35861 \\
0.8333 & 1.38359 & 1.38398 & 1.37985 & 1.37619 & 1.37462 & 1.36932 & 1.36463 & 1.36114 \\
0.8750 & 1.38570 & 1.38621 & 1.38209 & 1.37849 & 1.37696 & 1.37167 & 1.36696 & 1.36348 \\
0.9167 & 1.38768 & 1.38828 & 1.38420 & 1.38068 & 1.37916 & 1.37389 & 1.36915 & 1.36566 \\
0.9583 & 1.38956 & 1.39024 & 1.38622 & 1.38270 & 1.38125 & 1.37600 & 1.37121 & 1.36772 \\
1.0000 & 1.39168 & 1.39222 & 1.38797 & 1.38463 & 1.38343 & 1.37824 & 1.37302 & 1.36959 \\
\hline & & & & & & & &
\end{tabular}

Los volúmenes molares aparentes $\left({ }_{1}^{\varnothing} V\right)$ para $\left[\mathrm{Emim}^{+}\right]\left[\mathrm{CF}_{3} \mathrm{SO}_{3}{ }^{-}\right]$se calcularon a partir de los datos de densidad usando la ecuación (Yang et al., 2005).

${ }_{1}^{\emptyset} V=\left[1000\left(\rho_{0}-\rho\right)+m M_{L I} \rho_{0}\right] / m \rho \rho_{0}$

Donde $\rho_{0}$ y $\rho$ son las densidades del agua pura y la de la solución respectivamente, $m$, es la molalidad y $M_{L I}$ es la masa molar de $\left[\mathrm{Emim}^{+}\right]\left[\mathrm{CF}_{3} \mathrm{SO}_{3}{ }^{-}\right]$. En la tabla 4, se muestran simultáneamente el volumen molar aparente calculado a partir de la ecuación (5) y el volumen molar del líquido iónico puro. Aquí se observa que los volúmenes molares aparentes de la mezcla binaria [Emim+ $]\left[\mathrm{CF}_{3} \mathrm{SO}_{3}{ }^{-}\right]+$agua aumentan con el incremento de la temperatura.

Por otra parte, de acuerdo con las ecuaciones de trabajo (1-4) correspondientes a los modelos previamente

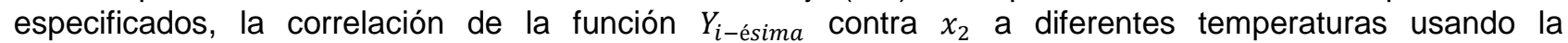
herramienta Solver del programa Excel, produce los parámetros de interacción y la desviación estándar que se muestran en la Tabla 5. Se destaca que la evaluación de estos parámetros se logró por minimización de la función objetivo:

$F . O=\sum_{1}^{n} \frac{\left({ }_{1}^{\phi} \mathrm{V}_{\text {exp }}-{ }_{1}^{\phi} \mathrm{V}_{\text {calc }}\right)^{2}}{n-p}$

Donde $n$ y $p$ corresponden al número de datos experimentales y el número de parámetros respectivamente. Los valores de los parámetros de la ecuaciones (1-4), la desviación estándar (s) y el valor del coeficiente de correlación $(r)$, se muestran en la Tabla 5. De acuerdo a los valores de $r$ mostrados en esta Tabla, el modelo de PS que usa como estado de referencia el soluto a dilución infinita se ajusta mejor a los ${ }_{1}^{\phi} V$ experimentales. 
Tabla 4: Volúmenes molares aparentes de $\left[\mathrm{Emim}^{+}\right]\left[\mathrm{CF}_{3} \mathrm{SO}_{3}{ }^{-}\right]+$agua a temperaturas de $283.15 \mathrm{~K}$ a $318.15 \mathrm{~K}$

\begin{tabular}{|c|c|c|c|c|c|c|c|c|}
\hline $\mathrm{T} / \mathrm{K}$ & 283.15 & 288.15 & 293.15 & 298.15 & 303.15 & 308.15 & 313.15 & 318.15 \\
\hline$X_{\mathrm{LI}}$ & \multicolumn{8}{|c|}{ 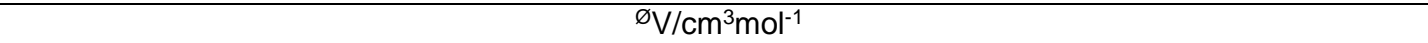 } \\
\hline 0.0417 & 184.74 & 185.54 & 186.48 & 187.56 & 188.11 & 189.38 & 190.09 & 191.35 \\
\hline 0.0860 & 185.40 & 186.06 & 186.95 & 187.84 & 188.48 & 189.52 & 190.24 & 191.32 \\
\hline 0.1250 & 185.78 & 186.35 & 187.20 & 188.04 & 188.63 & 189.63 & 190.35 & 191.29 \\
\hline 0.1667 & 186.07 & 186.56 & 187.37 & 188.18 & 188.71 & 189.67 & 190.48 & 191.22 \\
\hline 0.2065 & 186.28 & 186.70 & 187.48 & 188.26 & 188.74 & 189.67 & 190.50 & 191.14 \\
\hline 0.2500 & 186.46 & 186.81 & 187.56 & 188.31 & 188.74 & 189.65 & 190.44 & 191.06 \\
\hline 0.2917 & 186.59 & 186.89 & 187.61 & 188.33 & 188.74 & 189.61 & 190.38 & 190.97 \\
\hline 0.3333 & 186.69 & 186.94 & 187.65 & 188.34 & 188.72 & 189.58 & 190.32 & 190.89 \\
\hline 0.3750 & 186.77 & 186.98 & 187.68 & 188.34 & 188.69 & 189.53 & 190.26 & 190.82 \\
\hline 0.4167 & 186.84 & 187.01 & 187.69 & 188.34 & 188.66 & 189.49 & 190.20 & 190.75 \\
\hline 0.4583 & 186.90 & 187.04 & 187.70 & 188.32 & 188.63 & 189.44 & 190.15 & 190.68 \\
\hline 0.5000 & 186.94 & 187.05 & 187.70 & 188.31 & 188.60 & 189.40 & 190.09 & 190.62 \\
\hline 0.5417 & 186.98 & 187.06 & 187.70 & 188.29 & 188.57 & 189.35 & 190.04 & 190.56 \\
\hline 0.5833 & 187.01 & 187.07 & 187.69 & 188.26 & 188.53 & 189.31 & 189.99 & 190.50 \\
\hline 0.6250 & 187.04 & 187.07 & 187.68 & 188.24 & 188.50 & 189.27 & 189.94 & 190.44 \\
\hline 0.6667 & 187.05 & 187.07 & 187.67 & 188.22 & 188.46 & 189.22 & 189.89 & 190.39 \\
\hline 0.7083 & 187.07 & 187.06 & 187.65 & 188.19 & 188.43 & 189.18 & 189.84 & 190.34 \\
\hline 0.7500 & 187.08 & 187.05 & 187.64 & 188.16 & 188.39 & 189.14 & 189.80 & 190.29 \\
\hline 0.7917 & 187.08 & 187.04 & 187.62 & 188.13 & 188.35 & 189.09 & 189.75 & 190.24 \\
\hline 0.8333 & 187.08 & 187.03 & 187.60 & 188.10 & 188.31 & 189.05 & 189.71 & 190.19 \\
\hline 0.8750 & 187.08 & 187.01 & 187.57 & 188.06 & 188.27 & 189.00 & 189.66 & 190.15 \\
\hline 0.9167 & 187.07 & 186.99 & 187.54 & 188.02 & 188.23 & 188.96 & 189.61 & 190.10 \\
\hline 0.9583 & 187.05 & 186.96 & 187.51 & 187.98 & 188.18 & 188.90 & 189.56 & 190.05 \\
\hline $1.0000\left(\mathrm{~V}^{0} \mathrm{LI}\right)$ & 186.99 & 186.92 & 187.49 & 187.94 & 188.10 & 188.81 & 189.53 & 190.01 \\
\hline
\end{tabular}

Tabla 5: Parámetros de Pitzer-Simomson y Pitzer-Simomson-Clegg.

\begin{tabular}{|c|c|c|c|c|c|c|c|c|}
\hline$T / K$ & 283.15 & 288.15 & 293.15 & 298.15 & 303.15 & 308.15 & 313.15 & 318.15 \\
\hline \multicolumn{9}{|c|}{ Parámetros de Pitzer-Simomson, estado de referencia soluto a dilución infinita } \\
\hline a0.102 & 3.883 & 3.834 & 3.787 & 3.743 & 3.694 & 3.657 & 3.611 & 3.576 \\
\hline$W^{v} .10^{4}$ & -2.375 & -0.172 & 0.838 & 2.182 & 3.268 & 4.355 & 4.671 & 6.317 \\
\hline $\mathrm{U}^{\mathrm{v}} .10^{4}$ & 5.359 & 3.911 & 3.263 & 2.289 & 1.698 & 0.415 & 0.770 & -2.087 \\
\hline s. $10^{6}$ & 1.49 & 1.12 & 0.88 & 0.69 & 0.78 & 0.85 & 1.54 & 1.06 \\
\hline $\mathrm{r}$ & 0.999 & 0.998 & 0.998 & 0.997 & 0.997 & 0.997 & 0.994 & 0.999 \\
\hline \multicolumn{9}{|c|}{ Parámetros de Pitzer-Simomson, estado de referencia líquido iónico puro } \\
\hline$W^{v} .10^{4}$ & -3.129 & -0.505 & 0.936 & 1.961 & 3.014 & 3.014 & 3.014 & 6.015 \\
\hline $\mathrm{Uv}^{\mathrm{v}} .10^{4}$ & 7.487 & 4.785 & 2.864 & 3.049 & 3.783 & 3.783 & 0.234 & -0.799 \\
\hline s. $10^{6}$ & 23.42 & 10.60 & 4.63 & 4.53 & 12.92 & 19.74 & 4.63 & 4.70 \\
\hline $\mathrm{r}$ & 0.997 & 0.998 & 0.999 & 0.997 & 0.948 & 0.881 & 0.992 & 0.991 \\
\hline \multicolumn{9}{|c|}{ Parámetros de Pitzer-Simomson-Clegg, estado de referencia líquido iónico puro } \\
\hline$W^{v} .10^{4}$ & -4.512 & -1.296 & 0.731 & 1.949 & 2.022 & 3.282 & 4.525 & 6.639 \\
\hline $\mathrm{U}^{\mathrm{v}} \cdot 10^{4}$ & -68.282 & -33.273 & 2.967 & -9.185 & -45.789 & -48.451 & 0.369 & -1.107 \\
\hline$V^{V} .10^{4}$ & 99.465 & 50.421 & 0.858 & 15.040 & 63.533 & 64.421 & 1.182 & -2.627 \\
\hline $\mathrm{B}^{\vee} .10^{4}$ & 56.138 & 28.233 & -0.001 & 8.989 & 36.543 & 38.113 & 0.001 & 0.003 \\
\hline s. $10^{6}$ & 12.82 & 4.99 & 4.42 & 1.51 & 7.44 & 9.47 & 4.22 & 1.91 \\
\hline$r$ & 0.994 & 0.997 & 0.999 & 0.994 & 0.979 & 0.968 & 0.994 & 1.000 \\
\hline \multicolumn{9}{|c|}{ Parámetros de Pitzer-Simomson-Clegg simplificada, estado de referencia líquido iónico puro } \\
\hline$W^{v} .10^{4}$ & -1.063 & 0.439 & 0.733 & 2.501 & 2.501 & 5.624 & 4.522 & 4.522 \\
\hline $\mathrm{U}^{\mathrm{v}} .10^{4}$ & 6.479 & 4.324 & 2.963 & 2.785 & 2.785 & 2.304 & 0.373 & -1.101 \\
\hline$V^{\mathrm{V}} .10^{4}$ & -8.718 & -3.982 & 0.855 & -2.280 & -2.280 & -9.028 & 1.192 & -2.617 \\
\hline s. $10^{6}$ & 18.63 & 8.38 & 4.42 & 2.55 & 11.48 & 13.16 & 4.22 & 1.91 \\
\hline$r$ & 0.984 & 0.989 & 0.999 & 0.994 & 0.926 & 0.947 & 0.994 & 1.000 \\
\hline
\end{tabular}

Tabla 6: Valores de $t_{c a l}$ para comparar los volúmenes molares aparentes experimentales y calculados.

\begin{tabular}{lllllllll}
\multicolumn{10}{c}{$t_{c}=2.0739, \mathrm{p}=0.05, \mathrm{gl}=22$} \\
\hline \multicolumn{1}{c}{ T/K } & 283.15 & 288.15 & 293.15 & 298.15 & 303.15 & 308.15 & 313.15 & 318.15 \\
\hline PS dilución infinita & 0.0376 & 0.0310 & 0.0375 & 0.0463 & 0.0398 & 0.0359 & 0.0194 & 0.0284 \\
PS LI puro & 0.0016 & 0.0034 & 0.0080 & 0.0046 & 0.5522 & 0.0044 & 0.0035 & 0.0028 \\
PSC LI puro & 0.0111 & 0.0401 & 0.0709 & 0.0388 & 0.0132 & 0.0095 & 0.0142 & 0.0845 \\
PSCS LI puro & 0.0060 & 0.0025 & 0.0174 & 0.0092 & 0.0019 & 0.0039 & 0.0078 & 0.0524 \\
\hline
\end{tabular}


A fin de comparar los volúmenes molares aparentes calculados con los experimentales, en este artículo se muestran los resultados de la prueba $t$-student (Miller et al., 2002), tal como se aprecia en la Tabla 6. Debido a que $t_{\text {cal }}$ resultó menor que $t_{c}$ en todos los casos, se concluye que los datos comparados no presentan diferencias significativas con una confianza del $95 \%$. Un examen cuidadoso de los resultados obtenidos con el modelo de PS que usa como estado de referencia el soluto a dilución infinita, revela que las contribuciones de largo alcance (ión-molécula), contenidas en el término $\bar{V}_{L I}^{0} / 2 R T=a_{0}$ determinan el comportamiento volumétrico de estas soluciones; ya que este término es mucho mayor que los parámetros $W_{1, M X}^{V}$ y $U_{1, M X}^{V}$. Cabe resaltar que este tipo de análisis sería difícil de lograr si el modelo examinado usa al líquido iónico puro como estado de referencia. Por tanto es evidente que el primer modelo es mucho más recomendable para análisis de interacciones.

\section{CONCLUSIONES}

En este trabajo se reportan las densidades y los volúmenes molares aparentes obtenidos a partir de las medidas de densidad del sistema binario $\left[\mathrm{Emim}^{+}\right]\left[\mathrm{CF}_{3} \mathrm{SO}_{3}{ }^{-}\right]+$agua en el intervalo de concentración [0.0417 0.9583] en la escala de fracción molar a las temperaturas desde $283.15-318.15 \mathrm{~K}$. Se encontró que el modelo de PS que usa como estado de referencia el soluto a dilución infinita se ajusta mejor a los ${ }_{1}^{\phi} V$ experimentales y adicionalmente se encontró que las interacciones ión-molécula de largo alcance dominan el comportamiento volumétrico de la solución. También se infiere que el modelo de PS que usa como estado de referencia al líquido iónico puro, PSC y PSCs sólo podría usarse para caracterizar el comportamiento volumétrico de estos sistemas. Finalmente, la comparación de los volúmenes molares aparentes calculados con los diferentes modelos y los obtenidos experimentalmente, mostró que no presentan diferencias significativas con una confianza del $95 \%$, ya que los resultados de la prueba $t$-student, indicó que $t_{c a l}$ es menor que $t_{c}$ en todos los casos.

\section{AGRADECIMIENTOS}

Los autores agradecen a la Universidad de Córdoba por el apoyo prestado para la realización de este trabajo.

\section{REFERENCIAS}

Clegg, S.L. y K.S. Pitzer, Thermodynamics of multicomponent, miscible, ionic system: generalized equations for symmetrical electrolytes, J. Phys. Chem: 96, 3513-3520 (1992).

Galleguillos, H., A. Serreño y L. Cisternas, Evaluación de parámetros de interacción iónica para modelos de disoluciones acuosas de electrolitos, Información Tecnológica: 9(6), 35-50 (1998).

Kodejš, Z. y P. Pacák, Volumetric properties of concentrated electrolyte solutions, Chem. Papers: 40 (4) 523-535 (1986).

Miller, N.J. y J.C. Miller, Estadística y quimiometría para química analítica, 4a edición, 40-50, Pearson Educación, Madrid, España (2002).

Páez M.S., M.K.Vergara y E. Montes, Parámetros de interacción volumétricos del sistema 1-butil-3-metil imidazolio trifluorometanosulfonato + agua a varias temperaturas. Información Tecnológica: 24(6), 47-58 (2013).

Pitzer, K.S., Activity Coefficients in Electrolyte Solution, $2^{\text {a }}$ edición, capítulo 3, CRC Press: Boca Raton, FL (1991).

Pitzer, K.S. y J.M. Simonson, Thermodynamics of multicomponent, miscible, ionic systems: theory and equations, J. Phys. Chem: 90, 3005-3009 (1986).

Rodríguez, H. y J.F. Brennecke, Temperature and Composition Dependence of the Density and Viscosity of Binary Mixtures of Water + Ionic Liquid, J. Chem. Eng. Data: 51, 2145-2155 (2006).

Rowland, D. y P.M. May, A Pitzer-based characterization of aqueous magnesium chloride, calcium chloride and potassium iodide solution densities to high temperature and pressure, Fluid Phase Equilibia: 338, 5462 (2013).

Song, G.J., Properties of room temperature ionic liquid 3-ethyl-1-methylimidazolium ethyl sulfate, ЖУРНАЛ ФИЗИЧЕСКОЙ ХИМИИ: 84(5c), 859-864 (2010). 
Tong, J., Q.S. Liu, W. Guan y J.Z. Yang, Studies on volumetric properties of concentrated aqueous amino acid ionic liquid [ $C_{3}$ mim][Glu], J. Chem. Eng. Data: 54, 1110-1114 (2009).

Vercher, E. y A.V. Orchillés, P.J. Miguel y A. Martínez-Andreu, Volumetric and Ultrasonic studies of 1-ethyl3-methylimidazolium trifluoromethanesulfonate ionic liquid with methanol, ethanol, 1-propanol, and water at several temperatures, J. Chem. Eng. Data: 52, 1468-1482 (2007).

Wijesinghe, A. y J. Rard, Conversion and optimization of the parameters from an extended form of the ioninteraction model for $\mathrm{Ca}\left(\mathrm{NO}_{3}\right)_{2}(\mathrm{aq})$ and $\mathrm{NaNO}_{3}(\mathrm{aq})$ to those of the standard Pitzer model, and an assessment of the accuracy of the parameter temperature representations, J. Chem. Thermodynamics: 37, 1196-1218 (2005).

Yang, J.Z., X.M. Lu , J.S. Gui , W.G. Xu y H.W. Li, Volumetric properties of room temperature ionic liquid 2 The concentrated aqueous solutions of $\{1$-methyl-3-ethylimidazolium ethyl sulfate + water $\}$ in a temperature range of $278.2 \mathrm{~K}$ to $338.2 \mathrm{~K}$, J. Chem. Thermodynamics: 37, 1250-1255 (2005).

Zhang, Q.G., F. Xue, W. Guan, J. Tong y B. Wang, Studies on volumetric properties of concentrated aqueous solution of ionic liquid $\mathrm{BMIBF}_{4}$, J. Solution Chem: 35, 297-309 (2006). 
